\title{
Design and Analysis of Clustering Routing Protocol in Wireless Sensor Network of Frozen Food
}

\author{
https://doi.org/10.3991/ijoe.v13i12.7893 \\ Jianjun Wu, Xiao Feng, Hui-dang Zhang, Wei Lv( $\left.{ }^{(}\right)$ \\ Henan University of Technology, Zhengzhou, China \\ popp_ly@163.com
}

\begin{abstract}
In order to address the issues of uneven energy dissipation and uniform cluster coverage in wireless sensor networks (WSNs) of frozen food, we developed a load balancing and uniform coverage clustering (LBUCC) algorithm to find an efficient way to generate clusters. Considering node density and the coverage radius of cluster heads, the nearest neighbor clustering algorithm was adopted to cluster the sensor network. On the basis of the number of neighbors and the residual energy of nodes, the LBUCC algorithm ensures the equal distribution of cluster head responsibility among sensor nodes and performs well in periodic data gathering with selected cluster head. As the storage and distribution of frozen food is frequent, the clustering strategy was proposed for dynamic topology in this paper. The LBUCC algorithm was compared with LEACH-C and DHAC algorithms which are well-known in using centralized control algorithm to select cluster head. The simulation results demonstrate that the LBUCC algorithm has longer network lifetime and uniform coverage than the clustering protocols LEACH-C and DHAC do.
\end{abstract}

Keywords-Frozen food, Wireless sensor networks, Uniform clustering, even energy dissipation

\section{Introduction}

Fast-frozen food is the food that can be preserved long at $-18{ }^{\circ} \mathrm{C}$ or lower after prefreezing preparation at $-30{ }^{\circ} \mathrm{C} \sim-40^{\circ} \mathrm{C}$ in an equipment. In modern freezing technology, the food temperature can be reduced to below its congealing point in the shortest possible time, with all or most of moisture in the food turning into ice crystal, so that there will be insufficient liquid water for microorganisms and endoenzyme to work. Moreover, the lower storage temperature can ensure the quality of food during the freezing process and reserve the original freshness, color, flavor and nutrition of the food.

Fast-frozen food is maintained at a desired low-temperature range during production, storage, transportation and selling, which is called cold chain. The unbroken cold chain is vital to the quality of fast-frozen food. At home, the cold-chain equipment serving for food product manufacturing and logistics are independent of each other, connecting to different networks. As a result, the cold chain is interrupted at times, 
which is far below the standard of modern low-temperature logistics. Under the influence of various factors in cold chain designing, electric power supply, environmental change, etc., the temperature in cold storage is not always constant and thus damages the quality of frozen food. Therefore, it is far from enough by relying on cold chain system to monitor the temperature in fast-frozen food center. WSNs are effective to solve this problem with the objective data acquisition of temperature, humidity, location, etc. Regarding single-packed frozen food as one node of WSN, a large scale wireless sensor network will be built in frozen food storage and transportation. Given that the energy of WSN nodes is limited, it is of great significance to design a set of low energy consumption routing algorithms.

\section{Related Research}

At present, clustering routing algorithm with good extendibility is adopted in large scale WSNs; low energy adaptive clustering hierarchy protocol has matured into a typical hierarchical routing algorithm [1]. Under the LEACH protocol, the cluster is self-organized and the cluster head is randomly formed by some node. There is no interference between cluster members in CDMA communication system. Cluster members send data to cluster heads within their own allocated time slots in the TDMA frame; after receiving all these data, cluster heads fuse them and send the fused data to the far-off sink node. In this process, cluster heads have heavier workload and higher energy consumption than cluster members do. Operating in rounds, LEACH protocol creates clusters and transmits data in sequence in every round. Cluster creation is mainly about choosing cluster heads and creating clusters correspondingly. Data transmission includes the transmission from cluster member to cluster head and from cluster head to sink node.

How to generate cluster heads is the focus in researches on cluster-based routing algorithm. Taking LEACH protocol for example, as the cluster head is chosen from nodes according to node number, the number of cluster heads changes greatly in each round, which means uncontrollable distribution of cluster heads. However, this problem can be well solved by choosing cluster heads based on the global information in LEACH-C [2]. First, each node reports to the base station its geographical location and energy level; then, the base station averages current node energy and chooses nodes with higher residual energy than the average level as cluster heads. The base station adopts simulated annealing algorithm (SAA) to choose the best set of nodes to be the cluster heads from the candidate cluster sets. Finally, the base station reports the cluster head sets and cluster structure to complete network clustering. LEACH-C adopts collective control strategy, in which single-hop network is adopted by intra cluster and inter cluster data transmission due to network clustering. BCDCP gives labels [3] to clusters in order to evenly distribute network energy, in which two farthest nodes are selected as cluster heads among all candidate clusters and then the network is divided into two big sub-clusters, each of which has a suitable number of cluster members; next, the two sub-clusters are divided into $2^{\mathrm{n}}$ cluster members until reaching the expected number. In the labeling process, even energy dissipation and 
balanced cluster loads can be realized by maximizing the distance between cluster heads and equalizing cluster members, respectively. In every round, the base station chooses the candidate cluster head based on the average residual energy of nodes. Optimization is a must to choose one set of nodes as the final cluster head from the candidate cluster heads, i.e. to choose the best way of network clustering. In document [4] and document [5] particle optimization algorithm and ant colony algorithm were adopted to choose one set of nodes as the final cluster head, with heavy calculation load and inaccurate result. In document [6], Li Chengyue et al. used the LEACH$\mathrm{T}$ protocol to choose cluster head, in which the node with the shortest time intervals is the most possible to be chosen as cluster head. In this way, the number of cluster heads in each round is suitable enough to effectively balance the energy of the whole network, but the energy consumption of the timer used to choose cluster heads cannot be neglected, plus with increased complexity. In document [7], the residual energy of nodes and the distance between nodes themselves and between nodes and the base station are taken into consideration by researchers to reduce energy consumption in the cluster-based routing algorithm. In document [8], single-hop of intra cluster information is required considering the detection environment of random distributed nodes and that the cluster members and cluster heads are chosen according to the information of every node in the clustering table. This algorithm has a large amount of calculation work and neglects the even distribution of node numbers in different intra-clusters. As a summary, the cluster head selection methods above are not fair enough and may cause uneven energy consumption of network with too many or too few nodes.

As per energy consumption, the cluster tree routing algorithm of wireless sensor networks may be divided into the following types:

1. self-organized cluster [9-11]: the sensors in WSN are clustered differently in different strategies. The head of every cluster fuses the received data into less amount and then transmits them to the sink node.

2. minimum spanning tree [12-15]: Based on sink, a minimum spanning tree is gained by a kind of measure in WSN. Normal node transmits data to sink in multi-hop, and the energy consumption of node is regarded as the cost of data transmission.

At present, in order to reduce the cost of data transmission, the tree routing algorithm has been completely used to reduce the energy consumption of RF rather than MPU in node.

The above clustering algorithms are all about reducing the nodes' energy consumption, balancing energy load, or extending network life cycle, which seems off target when the network characteristics change in different application environments. In view of the large number of nodes and their frequent births and deaths in wireless sensor network, the network nodes are divided into reasonable clusters whose head is selected in a way that balancing the energy consumption of each node. The purpose is to protect partial nodes from dying soon or, in other words, to extend the life cycle of each node. Thinking about this case, the LBUCC routing algorithm is adopted in this paper, which takes full consideration of the number of nodes in clusters, the distance between cluster heads and the coverage of sub-clusters across the detection area. 


\section{Wireless Sensor Network Mode of Fast-frozen Food}

\subsection{Problems of wireless sensor network of fast-frozen food}

With considerable nodes and collective storage, the wireless sensor network of fast-frozen food has a large quantity of redundant data. Thus, the following problems need to be taken into account during the design of WSN routing protocol for fastfrozen food:

1. Energy consumption balance: All nodes in wireless sensor network of fast-frozen food are needed from the beginning of the cold chain all the way to the table, and thus the node energy should be high enough to maintain the life of nodes in this process. The rotation mechanism of cluster-based algorithm and the balanced number of nodes within a cluster are important in energy consumption of fastfrozen food network.

2. Balanced distance between adjacent clusters and uniform cluster coverage: Wireless sensor network of fast-frozen food requires that clusters have to cover the whole monitoring area of fast-frozen food evenly. Thus, the coverage radius of each cluster needs to be considered and each cluster head can represent the real environment data.

3. Extensibility: The storage and distribution of fast-frozen food and other factors induce the changes of network topology. Therefore, routing protocol should be able to deal with the birth and death of nodes and other incidents.

\subsection{Network mode of fast-frozen food}

Refrigerator car and cold storage are closed cuboids. In this paper, assuming that the network is composed of $\mathrm{N}$ centralized deployment of sensor nodes and is distributed in a $\mathrm{L}^{*} \mathrm{M} * \mathrm{~K}$ closed cuboid. In order to monitor the temperature, humidity and other environmental information in where the nodes are, the network nodes should have the following characteristics:

- The sink node is located in the lateral network and its computation power and energy are unrestricted;

- The operation of storage, delivery, loading, unloading, etc. will change the topology structure of wireless sensor network;

- Sensor nodes have different initial energy because of different time of birth. Nodes can sense the residual energy and fuse data.

- Wireless transmitting power of nodes is controllable and adjustable based on the distance between nodes;

- A node can calculate its distance from the sink node according to the signal intensity of nodes.

The energy consumption model of communication system in WSN is shown in figure 1. 


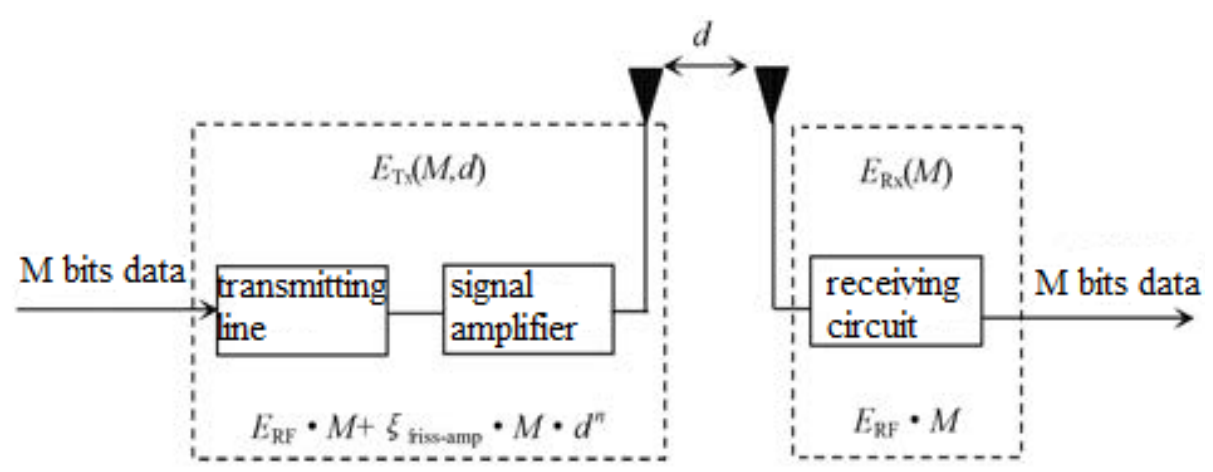

Fig. 1. Energy consumption model in wireless communication

This model includes the energy consumption in sending and receiving data. The energy consumed to send data is the energy consumed in the radio frequency module and signal amplifier; while the energy consumed to receive data is the energy consumed in electric circuit. $\xi_{\text {friss-amp }}$ represents the multiple of signal amplifier. The energy consumption in signal amplifier can be calculated by free-space and multi-path fading model according to the distance between each other. In fading model of free space, the value of path loss is 2 and $\mathrm{d}^{2}$ represents energy loss; in fading model of multi-path, the value of path loss is 4 and $d^{4}$ represents energy loss. $E_{R F}$ represents the energy consumption caused by sending or receiving $1 \mathrm{~b}$ electric circuit. Supposing that the signal path is symmetrical, the energy consumed in sending data from node 1 to node 2 is the same with the other way around. Based on the model shown in figure 1 , the energy consumption caused by sending $M$ bits data is shown in formula (1):

$$
E_{T X}(M, d)=\left\{\begin{array}{l}
E_{R F} \cdot M+\xi_{\text {friss-amp }} \cdot M \cdot d^{2}, d<d_{e} \\
E_{R F} \cdot M+\xi_{\text {friss-amp }} \cdot M \cdot d^{4}, d \geq d_{e}
\end{array}\right.
$$

In which $\xi_{\text {friss-amp }}$ is related with transmission exaggeration model; $d_{c}$ represents distance constant and can be expressed in formula (2);

$$
\mathrm{d}_{\mathrm{e}}=\frac{4 \pi \sqrt{\mathrm{L}_{\mathrm{S}}}}{\lambda} \mathrm{h}_{\mathrm{s}} \mathrm{h}_{\mathrm{l}}
$$

In formula (2), $\mathrm{L}_{\mathrm{S}}$ represents energy consumption of the system and is unrelated with the number of transmission data; $h_{S}$ represents the length of the receiving antenna of the node; $h_{1}$ represents the length of the sending antenna of the node; $\lambda$ represents carrier wave length.

The value of transmission distance is $\mathrm{d}$, and the energy consumption caused by receiving $\mathrm{M}$ bits data is shown in formula (3):

$$
\mathrm{E}_{\mathrm{RX}}(\mathrm{M})=\mathrm{E}_{\mathrm{RF}} \cdot \mathrm{M}
$$




\section{Routing Protocol Of Wireless Sensor Network of Fast-frozen Food}

Given the specific application environment of wireless sensor network formed in storage and refrigerator truck, the protocol adopts rotation mechanism similar to the one in document [2]. In each round are two phases: cluster creation and steady state stages. Clusters are created by using the centralized control strategy, in which the base station is responsible for network clustering; the routing within and across clusters is single-hop.

\subsection{Clustering creation}

In this protocol, clustering creation is completed in base station, which includes three steps: collecting node information, running the clustering algorithm to choose cluster heads and its members, and reporting clustering information.

1. Colleting node information: Given the specific application environment of wireless sensor network formed in storage and refrigerator truck, the base station informs each node to collect the adjacent node information. At this command, each node needs to look for its own adjacent node with power in radio frequency within a radius of $\mathrm{R}=\frac{\sqrt{2 \cdot \mathrm{S} / \mathrm{K}}}{2}$, in which $\mathrm{S}$ stands for the monitoring area, and $\mathrm{K}$ stands for the preset number of clusters. In creating the first cluster, nodes transmit the initial energy and the adjacent node information to base station.

2. Choosing cluster heads: On the basis of the residual energy and adjacent energy of nodes, cluster heads are chosen in the following steps:

(1) Choose nodes with residual energy higher than the value of the threshold $\mathrm{E}(\mathrm{r})$ to compose a set, where $\mathrm{r}$ represents the value of round;

(2) Choose adjacent nodes with similar expected number by cluster to join into the cluster set from the set of candidate clusters;

(3) Delete the information of node and the adjacent node from the set of candidate cluster;

(4) If it is required to empty the candidate set, repeat step 2 to 3 until the candidate set is empty.

3. Distributing clustering information: The base station generates clusters in a round with the node information of cluster heads.

(1) The nodes chosen as cluster heads can report information to the surrounding nodes. Nodes decide whether to join the cluster or not according to the signal strength to complete network clustering under the principle of proximity.

(2) TDMA progress chart is built by cluster head; and the data transmission time for each node is related to the number of nodes within a cluster. 


\subsection{Steady phase}

In this phase, there are three steps:

1. Cluster member transmitting data to cluster head: Cluster members send temperature data (including temperature data stored in register; the register becomes zero after data transmission) to the cluster head within its own time slot in the TDMA slot table and remain in latent periods at other times.

2. Data fusion: After receiving data from its member, the cluster head will provide initial judgment like temperature change across the preset warning line. It will also record the information of node number, temperature data, data collecting time, and the average temperature of other nodes, and fuse all the message before sending it to the base station.

3. Cluster head transporting data to base station: The base station collects information of each cluster head based on TDMA mechanism. In case the temperature of any node is above the warning line, the base station will write the corresponding node number, temperature data, and collection time into the data base, with the average cluster temperature of other nodes written into the central data base.

4. Adjustment phase: Network clustering can be carried out both in fixed time intervals and during network topology change. However, the following measures need to be taken for adjustment in different situation:

(1) Storage of fast-frozen food: when new nodes join in, the principle of proximity will be followed if the number of the nodes is fewer than the expected value by cluster; otherwise, the clustering situation should be readjusted by using the clustering algorithm;

(2) Distribution of fast-frozen food:

(1) if any node other than the cluster head dies while maintaining the clustering condition unchanged, the cluster head will send to the base station the information of those nodes that no more contact with it;

(2) If the cluster head dies, the network clustering situation should be readjusted by using the clustering algorithm.

\section{Protocol Emulation and Analysis}

Given that fast-frozen food is stored in cold storage and refrigerator truck in large centralized quantity, tens of thousands of nodes will be set in a fixed storage space and the node sink is located in the point $(0,0)$, which is the corner of cold storage or refrigerator truck. Supposing the initial energy of all nodes are $0.5 \mathrm{~J}$, we have

$\mathrm{E}_{\mathrm{elec}}=\frac{50 \mathrm{pJ}}{\mathrm{bit}}, \varepsilon_{\mathrm{fs}}=\frac{10 \mathrm{pJ}}{\mathrm{bit} \cdot \mathrm{m}^{-2}}, \varepsilon_{\mathrm{mp}}=\frac{0.013 \mathrm{pJ}}{\mathrm{bit} \cdot \mathrm{m}^{-4}}$. The information each node sending to the cluster head can be 2000 bits, and the energy consumption of information fusion can be calculated by the formula $E_{D A}=\frac{5 n J}{b i t}$. In order to verify the effectiveness of the LBUCC clustering algorithm applied to the wireless sensor network of fast-frozen food proposed in this paper, we compare this algorithm with the centralized control algorithms of LEACH-C and DHAC. 


\subsection{The time of the first death of nodes}

The nodes of wireless sensor network of fast-frozen food must be in effect, which means that nodes are not allowed to die within the validity period. Therefore, the number of rounds at the time when the first node dies is used to measure the survival period of network. Due to space limits, the total energy consumption of network in each round is listed in appendix 1. The total energy consumption of network in each round includes energy consumption in cluster creation and data transmission. Time intervals between each round can be decided according to the time internals for data collection required in real life. In the emulation process, the value of network nodes can be any one of the ten numbers $100,200,300, \ldots, 1,000$. To avoid the influence of random deployment, the task will be carried out five times in each situation, and the five numbers of rounds at the death of the first node will be averaged for comparison.

Figure 2 shows the number of rounds at the death of the first node by LBUCC algorithm, LEACH-C algorithm and DHAC algorithm, respectively. As can be seen, the proposed LBUCC algorithm has the longest survival time of network with the gradual increase of node numbers, indicating that this algorithm is the best one when the node number increases to the peak value. For the LBUCC algorithm, given that most of the WSN nodes are stored in a centralized way, the data of cluster head is representative enough of the environment condition within the cluster; also, if the energy consumed to fuse cluster data is avoided, the life cycle of cluster head will be further extended in practical application.

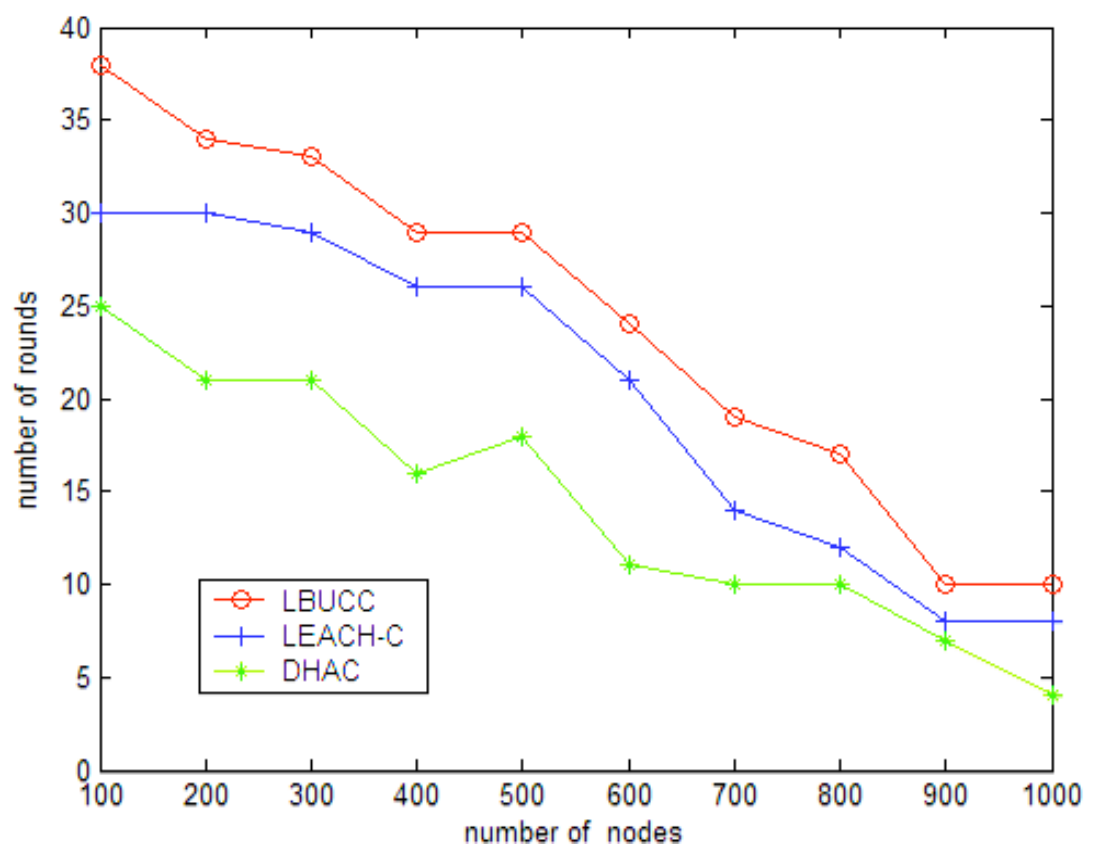

Fig. 2. Time Diagram of death of first nodes 


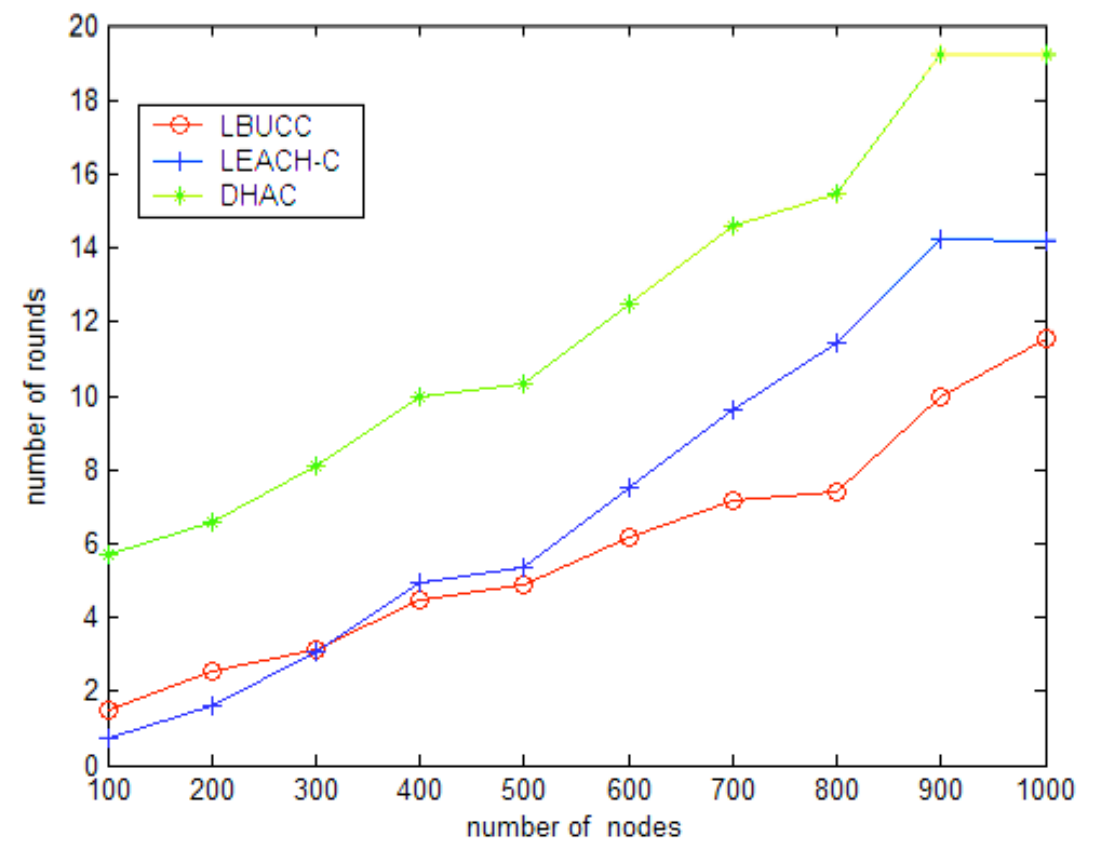

Fig. 3. Standard deviation of energy consumption of cluster heads

In another view, energy consumption balance of cluster head can reflect energy consumption balance of WSN nodes. Limited by the space, the analysis of energy consumption of cluster heads is shown in appendix 1. Energy consumption balance of cluster head can be calculated by standard deviation in formula (4):

$$
\mathrm{E}_{\mathrm{CH}}=\sum_{\mathrm{i}=1}^{\mathrm{k}}\left|\mathrm{E}_{\mathrm{i}}-\overline{\mathrm{E}}\right|
$$

In the formula, $\bar{E}=\sum_{i=1}^{k} E_{i} / k, k$ stands for the number of network clusters in each round, $E_{i}$ stands for the energy consumption of $i$, and $\bar{E}$ stands for the average consumed energy of cluster heads.

As shown in figure 3, when the number of node is bigger than 300 , the standard deviation of energy consumption of cluster heads is the lowest. It shows that LBUCC algorithm is suitable in the cases with a large number of nodes, because it can balance the number of cluster nodes and their distances from each other so that no cluster head dies earlier than the expected time.

\subsection{Evenness of cluster heads distribution}

In order to obtain the correct monitored temperature of fast-frozen food, it is required that each cluster head in wireless sensor network should exactly indicate the temperature within the cluster, and that the monitored temperature of fast-frozen food can be represented by the temperature recorded by all cluster heads. Therefore, the 
distribution evenness of cluster heads in network for frozen food in monitoring is used to represent the distribution of cluster heads.

$$
\mathrm{L}=\frac{4 \sum_{\mathrm{i}=1}^{\mathrm{k}} \pi \mathrm{r}^{2}}{\pi \mathrm{A}}
$$

In the formula, $\mathrm{r}_{\mathrm{i}}=\frac{1}{2} \min \left\{\mathrm{d}_{\mathrm{i}}\left(\mathrm{H}_{\mathrm{i}}, \mathrm{H}_{\mathrm{j}}\right)\right\}, \mathrm{d}_{\mathrm{i}}\left(\mathrm{H}_{\mathrm{i}}, \mathrm{H}_{\mathrm{j}}\right)$ stands for the Euclidean distance from cluster head $\mathrm{H}_{\mathrm{i}}$ to $\mathrm{H}_{\mathrm{j}}$, and $\mathrm{A}$ stands for the total area being monitored.

LBUCC algorithm has the highest distribution evenness of cluster heads, which means the most even distribution of cluster heads in monitoring area, followed by the DHAC algorithm and LEACH-C algorithm in the descending order. The main reason is that nodes are in centralized storage in the application background built in this paper, which facilitates the even distribution of clusters and the reduction in energy consumption; the standard deviation of the number of nodes within clusters by LBUCC algorithm is the lowest, which means the difference of the number of nodes within clusters is the lowest. The bigger the standard deviation is, the more unbalanced the number of nodes within clusters is. From another point of view, it indicates that the LBUCC algorithm is the most balanced one among all.

\section{Conclusions}

Considering the birth and death of WSN nodes and their large quantity and centralized storage for fast-frozen food, the LBUCC routing protocol is proposed in this paper and compared with DHAC and LEACH-C algorithms with respect to the network life cycle, the even coverage of the targeted cluster and other performance parameters. Experimental data shows that LBUCC protocol has the advantage of long life cycle of network, low consumption of energy, and even coverage of targeted cluster. In the follow-up study, to ensure that nodes of network will not die within the validity period, we need to further experiment on the energy consumption of network applied in cold storage and refrigerator truck by adopting LBUCC algorithm proposed in this paper combined with specific hardware and software environment.

\section{$7 \quad$ Acknowledgment}

Sincerely thank the Project supported by the Special Fund for Grain scientific, Research in the Public Interest (Grant No: 201413003), and Key scientific research project of Henan Province (Grant No: 280090), and Henan University of Technology, Doctor Fund (Grant No: 150575), and Open fund of key Laboratory of Grain Information Processing and Control (under Grant No.KFJJ-2015-103) 


\section{Appendix}

Calculate the total energy consumption of network in respective cluster creating and data transporting phases in each round.

\subsection{Energy consumption in the period of cluster creating:}

1) Energy consumption of node i by searching its adjacent node

$$
E_{\text {find }}(i)=\left(E_{\text {elec }}+\xi_{f s} \cdot R^{2}+\sum E_{\text {elec }} \cdot N_{i}\right) \cdot L_{c t l}
$$

In the formula, $N_{i}$ stands for the adjacent node of node I, $L_{a t l}$ stands for the number of bits of the information.

Total energy consumption of nodes of network by searching adjacent node can be calculated as:

$$
E_{\text {find }}=\sum_{i=1}^{N} E_{\text {find }}(i)
$$

In the formula, $N$ stands for the total number of nodes.

2) Energy consumption of node by sending data to the base station can be calculated as:

$$
E_{T x}(i)=E_{\text {elec }} \cdot I+\xi_{f s} \cdot I \cdot d_{i}^{2}
$$

In the formula, $I$ stands for the length of data package, $d_{i}$ stands for the distance from node $i$ to the base station.

Total energy consumption of nodes by sending data to the base station can be calculated as

$$
E_{T x}=\sum_{i=1}^{N} E_{T x}(i)
$$

3) Energy consumption of node by receiving information from the base station can be calculated as:

$$
E_{R x}=\sum_{i=1}^{N} E_{\text {elec }} \bullet I
$$

4) Energy consumption of node i by joining the cluster can be calculated as:

$$
E_{c l s}(i)=\sum_{j=1}^{K} E_{e l e c} \cdot L_{c t l}
$$


$K$ stands for the number of cluster heads

Total energy consumption of nodes by joining the cluster can be calculated as

$$
E_{c l s}=\sum_{i \notin H}^{N} E_{c l s}(i)
$$

$H$ stands for the set of cluster heads.

5) Energy consumption of cluster head i by searching for the cluster member can be calculated as:

$$
E_{\text {head }}(i)=E_{\text {elec }} \cdot L_{c t l}+\xi_{f s} \cdot L_{c t l} \cdot R^{2}
$$

Total energy consumption of all cluster heads by searching for cluster members can be calculated as:

$$
E_{\text {head }}=\sum_{i \in H} E_{\text {head }}(i)
$$

The total energy consumption of building cluster is the sum of the value in formula (2), (4), (5), (7) and (9), as shown in formula 10:

$$
E_{\text {totalF }}=E_{\text {find }}+E_{T x}+E_{R x}+E_{c l s}+E_{\text {head }}
$$

\subsection{Energy consumption of data transmission}

1) The energy consumption by sending data from the cluster member $j$ to its cluster head i can be calculated as:

$$
E_{T x}(i, j)=E_{e l e c} \bullet I+\xi_{f s} \bullet I \bullet d_{i j}^{2}
$$

In the formula, $d_{i j}$ stands for the distance from node $\mathrm{j}$ to cluster head $\mathrm{i}$.

2) Energy consumption by sending data of all members can be calculated as:

$$
E_{T x h}=\sum_{i=1}^{K} \sum_{j \in H_{i}} E_{T x}(i, j)
$$

3) Energy consumption by receiving data of cluster members form cluster head i can be calculated as:

$$
E_{R x}(i)=\sum_{j=1}^{M} E_{\text {elec }} \bullet I
$$

In the formula, $M$ stands for the number of cluster members. 
4) Energy consumption by receiving member data from all cluster heads of network can be calculated as:

$$
E_{R x h}=\sum_{i=1}^{K} E_{T x}(i)
$$

Energy consumption of data fusion of cluster head i can be calculated as:

$$
E_{d a}(i)=\sum_{j=1}^{M} \xi_{d a} \bullet I
$$

In the formula, $\xi_{d a}$ stands for the energy consumption by fusing 1 bite data.

Energy consumption by data fusion of all cluster heads can be calculated as:

$$
E_{d a}=\sum_{i=1}^{K} E_{d a}(i)
$$

Energy consumption by sending data from cluster head $i$ to the base station can be calculated as:

$$
E_{T x s}(i)=E_{e l e c} \cdot I+\xi_{f s} \cdot I \cdot d_{i s}^{2}
$$

Total energy consumption by sending data from all cluster heads to the base station can be calculated as:

$$
E_{T x S}=\sum_{i=1}^{K} E_{T x S}(i)
$$

In data transmission, the total energy consumption of sending data once is the sum of the value calculated in formula (12), (14), (16) and (18), i.e.

$$
E_{\text {tatals }}=E_{T x h}+E_{R x h}+E_{d a}+E_{T x S}
$$

To sum up, the total energy consumption of clustering in each round is the sum of the value calculated in formula (10) and (19), i.e.

$$
E_{\text {total }}=E_{\text {totalF }}+E_{\text {totals }}
$$

In the practical application of WSNs, since the node density usually is higher (for example, 20 nodes per square meter), there is no need that each node to be chosen as the cluster head, and the value of threshold $\mathrm{T}$ is set to control the proportion of candidate cluster heads. 
In data fusion model within clusters, it is supposed that cluster head receives data of $\mathrm{k}$ bits sent by each member and the energy consumption of data fusion is set as $\mathrm{ED}=5 \mathrm{n} \mathrm{J} /$ bit no matter how many nodes within the cluster need to be compressed in the unit of $\mathrm{K}$ bits.

\section{References}

[1] Heinzelman W., Chandrakasan A., and Balakrishnan H. (2000). Energy-Efficient Communication Protocol for Wireless Microsensor Networks. Proceedings of the 33rd Hawaii International Conference on System Sciences (HICSS), IEEE Computer Society Press, 8, 1$10 \mathrm{https} / / /$ doi.org/10.1109/HICSS.2000.926982

[2] Heinzelman W., Chandrakasan A., and Balakrishnan H. (2002). An Application-Specific Protocol Architecture for Wireless Microsensor Networks, IEEE Transactions on Wireless Communications, pp. 660-670. https://doi.org/10.1109/TWC.2002.804190

[3] Muruganathan S., Ma D., Bhasin R. (2005). A centralized energy-efficient routing protocol for wireless sensor networks, IEEE Communications Magazine, 43(3), pp. 8-13. https://doi.org/10.1109/MCOM.2005.1404592

[4] Latiff N.M.A., Tsimenidis C.C., Sharif B.S. (2007). Energy-aware clustering for wireless sensor networks using particle swarm optimization, IEEE 18th International Symposium on Personal,Indoor and Mobile Radio Communications. Athens, pp. 1-5.

[5] Ziyadi M., Yasami K., Abolhassani B. (2009). Adaptive clustering for energy efficient wireless sensor networks based on ant colony optimization, Seventh Annual Communication Networks and Services Research Conference, IEEE Computer Society, pp. 330-334. https://doi.org/10.1109/CNSR.2009.58

[6] Li C.Y., Shen X.J., Chen H.P. (2010). Research and improvement of LEACH routing algorithm in Wireless Sensor Networks, Chinese Journal of Sensors and Actuators, pp. 11631167.

[7] Gautam N., Pyun J.Y. (2010). Distance Aware Intelligent Clustering Protocol for Wireless Sensor Networks, Journal of Communications and Networks, pp. 122-129. https://doi.org/10.1109/JCN.2010.6391368

[8] Lung C.H., Zhou C. (2010). Using Hierarchical Agglomerative Clustering in Wireless Sensor Networks: An Energy-efficient and Flexible Approach. Ad Hoc Networks, pp. 328344. https://doi.org/10.1016/j.adhoc.2009.09.004

[9] Arora V.K., Sharma V., Sachdeva M. (2016). A survey on LEACH and other's routing protocols in wireless sensor network. OPTIK, pp. 6590-6600. https://doi.org/10.1016/ j.ijleo.2016.04.041

[10] Sabet M., Naji H. (2016). An energy efficient multi-level route-aware clustering algorithm for wireless sensor networks: A self-organized approach, Computers \& Electrical Engineering, pp. 399-4111. https://doi.org/10.1016/j.compeleceng.2016.07.009

[11] Guo W., Zhang B., Chen G. (2013). A PSO-Optimized Minimum Spanning Tree-Based Topology Control Scheme for Wireless Sensor Networks, International Journal Of Distributed Sensor Networks, (1), pp. 179-180. https://doi.org/10.1155/2013/985410

[12] Zhu Y.H., Wu W.D., Pan J. (2010). An energy-efficient data gathering algorithm to prolong lifetime of wireless sensor networks., Computer Communications, pp. 639-647. https://doi.org/10.1016/j.comcom.2009.11.008

[13] Kim T., Seo S.C., Kim D. (2015). Distributed formation of degree constrained minimum routing cost tree in wireless ad-hoc networks, Journal Of Parallel And Distributed Computing, pp. 143-158. https://doi.org/10.1016/j.jpdc.2015.05.006 
[14] Singh A., Sundar S. (2011). An artificial bee colony algorithm for the minimum routing cost spanning tree problem, Soft Computing, pp. 2489-2499. https://doi.org/10.1007/s00500-011-0711-6

[15] Bhattacharya A., Kumar A. (2014). A shortest path tree based algorithm for relay placement in a wireless sensor network and its performance analysis, Computer Networks, pp. 48-62. https://doi.org/10.1016/j.comnet.2014.06.011

\section{Authors}

Jianjun $\mathbf{W u}$ is associate professor at the School of Information Science and Engineering, Henan University of Technology, Zhengzhou, 450001, China, with the research fields of computer applications and computer information technology.

Huidang Zhang is professor at the School of Information Science and Engineering, Henan University of Technology, Zhengzhou, 450001, China, whose research field is computer applications.

Xiao Feng is associate professor at the School of Information Science and Engineering, Henan University of Technology, Zhengzhou, 450001, China, whose research field is computer applications.

Wei Lv is with the School of Information Science and Engineering, Henan University of Technology, Zhengzhou, 450001, China.

Article submitted 25 October 2017. Published as resubmitted by the authors 28 November 2017. 